\title{
Recurrence in traumatic anterior shoulder dislocations increases the prevalence of Hill-Sachs and Bankart lesions: a systematic review and meta-analysis
}

\author{
Cain Rutgers $^{1} \cdot$ Lukas. P. E. Verweij $^{2} \cdot$ Simone Priester-Vink $^{3} \cdot$ Derek F. P. van Deurzen $^{2} \cdot$ Mario Maas $^{4}$. \\ Michel P. J. van den Bekerom ${ }^{2}$
}

Received: 16 August 2021 / Accepted: 13 December 2021 / Published online: 6 January 2022

(c) The Author(s) 2022

\begin{abstract}
Purpose The extent of shoulder instability and the indication for surgery may be determined by the prevalence or size of associated lesions. However, a varying prevalence is reported and the actual values are therefore unclear. In addition, it is unclear whether these lesions are present after the first dislocation and whether or not these lesions increase in size after recurrence. The aim of this systematic review was (1) to determine the prevalence of lesions associated with traumatic anterior shoulder dislocations, (2) to determine if the prevalence is higher following recurrent dislocations compared to first-time dislocations and (3) to determine if the prevalence is higher following complete dislocations compared to subluxations.

Methods PubMed, EMBASE, Cochrane and Web of Science were searched. Studies examining shoulders after traumatic anterior dislocations during arthroscopy or with MRI/MRA or CT published after 1999 were included. A total of 22 studies (1920 shoulders) were included.

Results The proportion of Hill-Sachs and Bankart lesions was higher in recurrent dislocations (85\%; 66\%) compared to first-time dislocations $(71 \% ; 59 \%)$ and this was statistically significant $(P<0.01 ; P=0.05)$. No significant difference between recurrent and firsttime dislocations was observed for SLAP lesions, rotator-cuff tears, bony Bankart lesions, HAGL lesions and ALPSA lesions. The proportion of Hill-Sachs lesions was significantly higher in complete dislocations (82\%) compared to subluxations (54\%; $P<0.01)$. Conclusion Higher proportions of Hill-Sachs and Bankart were observed in recurrent dislocations compared to first-time dislocations. No difference was observed for bony Bankart, HAGL, SLAP, rotator-cuff tear and ALPSA. Especially when a Hill-Sachs or Bankart is present after first-time dislocation, early surgical stabilization may need to be considered as other lesions may not be expected after recurrence and to limit lesion growth. However, results should be interpreted with caution due to substantial heterogeneity and large variance.
\end{abstract}

Level of evidence IV.

Keywords Epidemiology $\cdot$ Prevalence $\cdot$ Shoulder instability $\cdot$ Shoulder dislocation $\cdot$ Glenoid labrum $\cdot$ Labrum lesions . Hill-Sachs

Cain Rutgers

c.rutgers@student.vu.nl

1 Vrije Universiteit Amsterdam, De Boelelaan 1105, 1081 HV Amsterdam, The Netherlands

2 Shoulder and Elbow Unit, Joint Research, Department of Orthopaedic Surgery, OLVG, Amsterdam, The Netherlands

3 Medical Library, Department of Research and Epidemiology, OLVG, Amsterdam, The Netherlands

4 Division of Musculoskeletal Radiology, Department of Radiology and Nuclear Medicine, Amsterdam UMC, Location AMC, University of Amsterdam, Amsterdam, The Netherlands

\author{
Abbreviations \\ PRISMA Preferred Reporting Items for Systematic \\ Reviews and Meta-Analyses \\ JBI Joanna Briggs Institute \\ CI Confidence interval \\ SD Standard deviation
}

\section{Introduction}

Anterior shoulder dislocations commonly occur following a fall or direct impact to the shoulder at home or during sports/recreation activities, reporting an incidence of 
23.9 per 100,000 person-years and making up $45 \%$ of all dislocations [1-3]. Over $95 \%$ of dislocations occur in the anterior direction, with the other $5 \%$ occurring in the posterior or inferior direction [4].

Traumatic anterior shoulder dislocations are often accompanied by lesions of the soft-tissue and bony structures in and around the glenohumeral joint [5]. Soft-tissue lesions are typically diagnosed through imaging, such as MRI or MRA, bony lesions through CT or CTA and ligamentous lesions through CTA or MRA. Compared to the gold standard arthroscopy, the sensitivity of imaging techniques varies between 60 and $95 \%$ soft-tissue lesions while CTA shows sensitivity and specificity close to $100 \%$ for bony lesions. [6-10]

Evidence regarding the prevalence of lesions is lacking. Systematic reviews determining the prevalence are limited and epidemiological studies show a varying range in the observed prevalence of lesions [11-13]. Multiple variables may attribute to this disagreement, such as the experience of the assessor, machine settings [14], accuracy of diagnostic tools [15], selection bias and unclear differentiation between first-time or recurrent $(\geq 2)$ dislocation or subluxation [16]. A lack in epidemiological knowledge is problematic because the optimal management can vary between lesions. Little glenoid bone loss and low risk of recurrence may indicate a Bankart repair, whereas high glenoid bone loss and high risk of recurrence may indicate bony reconstruction such as the Latarjet procedure [17, 18]. Delayed surgical intervention, when this is needed, may cause a higher recurrence rate, degenerative changes and an increase in symptoms, especially in overhead and contact sports athletes [19-21]. Some lesions are more likely related to these outcomes than others, such as bony lesions, which are thought to be a risk factor for failure of conservative management and after soft-tissue stabilization [21,22]. This makes the presence and size of bony lesions important for surgical decision-making. Epidemiological knowledge of associated lesions may allow professionals to anticipate on these lesions and assist in deciding on the optimal management [23-27].

Recent literature shows a paradigm shift from a more conservative approach to earlier surgical intervention to prevent recurrence and increase shoulder function [21, 28]. The presence and size of lesions may influence the extent of instability and determine the technique of surgical shoulder stabilization. Therefore, the aim of this systematic review was (1) to determine the prevalence of lesions associated with traumatic anterior shoulder dislocations, (2) to determine if the prevalence is higher following recurrent dislocations compared to first-time dislocations and (3) to determine if the prevalence is higher following complete dislocations compared to subluxations. It was hypothesized that lesions were more prevalent following recurrent dislocations compared to first-time dislocations and following complete dislocations compared to subluxations.

\section{Materials and methods}

This systematic literature review was written according to the Preferred Reporting Items for Systematic Reviews and Meta-Analyses (PRISMA) statements, extended with a reporting standard to fit the needs for a systematic literature review [23]. This review has been registered to the Prospective Register of Systematic Reviews (PROSPERO registration ID: CRD42021233391, date of submission: 26-01-2021) [29].

\section{Search strategy}

Relevant studies were identified by searching PubMed, Embase/Ovid, Cochrane Database of Systematic Reviews/ Wiley, Cochrane Central Register of Controlled Trials/ Wiley, and Web of Science/Clarivate Analytics by C.R. and S.P.V. The search was started and completed on December 17,2020 . The following terms, including synonyms and closely related words, were used as index terms or free-text words: 'Shoulder dislocation', 'Arthroscopy', 'MRI', 'CT' and terms for all included lesions. A filter was applied to find articles published from 2000 until at least the end of 2020. The complete search strategies can be found in Additional file 1.

\section{Study selection and quality assessment}

Studies were independently assessed for eligibility by 2 authors (C.R. and L.P.E.V.), who individually screened the titles and abstracts using Rayyan (Hamad Bin Khalifa University, Doha, Qatar) [30]. Eligible studies were included in the full-text screening, which was also performed individually by 2 authors (C.R. and L.P.E.V.). Studies that met the inclusion criteria were included for analysis. Disagreement between authors was resolved by discussion and consensus.

Studies reporting the prevalence of associated lesions following traumatic anterior shoulder dislocations diagnosed lesions through arthroscopy, MRI, MRA, CT or CTA in a cohort of at least 10 patients were included. Studies written in English, Dutch or German were included. All cohorts had to be studied after the year 2000 due to the rapid progression of accuracy of diagnostic tools [31]. When case cohorts of two separate articles were overlapping, the article with the most different types of lesions was included. When this was the same between 
two articles, the article with the largest sample size was included. Cohort studies that followed patients after an intervention were only included when the prevalence of lesions for the entire population at the start of the followup period was reported.

Reviews, case reports, meta-analyses, biomechanical studies and cadaveric studies were excluded. Studies measuring the accuracy of diagnostic tools were excluded to prevent selection bias.

\section{Risk of bias}

The risk of bias was independently assessed by 2 authors (C.R. and L.P.E.V.) using the Joanna Briggs Institute (JBI) standard for critical appraisal. This tool was designed for systematic reviews reporting prevalence data [32]. Disagreement between authors was resolved by discussion and consensus.

\section{Identification of lesions}

Arthroscopy was considered the gold standard for all lesions. When lesions were not identified by arthroscopy, MRI or MRA was considered the highest ranking modality for softtissue lesions and CT or CTA was considered the highest ranking modality for bony lesions.

\section{Data extraction}

Baseline characteristics included sample size, male/female ratio, mean age with range and standard deviation (SD), indication for inclusion (e.g., arthroscopic surgery), highest ranking modality and population characteristics (e.g., athletes or military personnel). The primary outcome was the prevalence of lesions associated with traumatic anterior shoulder dislocations. The included lesions were defined in Table 1 in cooperation with an experienced musculoskeletal radiologist (M.M.) and orthopedic surgeon that specializes in shoulder and elbow pathology (M.P.J.B.). The authors

Table 1 Included lesions and definitions

\begin{tabular}{|c|c|}
\hline Lesions & Definition \\
\hline \multicolumn{2}{|l|}{ Bony lesions } \\
\hline Hill-Sachs & Impression fracture of the posterolateral humeral head \\
\hline Glenoid lesion & Depressed or raised surface of the glenoid \\
\hline Loose bodies & $\begin{array}{l}\text { A loose osseous fragment inside the glenohumeral joint, originating } \\
\text { from the glenoid rim or humeral head }\end{array}$ \\
\hline Bony Bankart & A bony lesion or fracture involving the anterior labrum and glenoid rim \\
\hline \multicolumn{2}{|l|}{ Soft-tissue lesions } \\
\hline Bankart & A tear of the anterior labrum at $2-6$ o'clock position \\
\hline Posterior Bankart & A tear of the posterior labrum at $6-11$ o'clock \\
\hline Perthes & $\begin{array}{l}\text { Anteroinferior labrum is partially detached and periosteum is stripped } \\
\text { medially but still intact }\end{array}$ \\
\hline Anterior Labral Periosteal Sleeve Avulsion (ALPSA) & $\begin{array}{l}\text { The labroligamentous complex is displaced medially; however, the } \\
\text { labrum and glenoid rim are still intact }\end{array}$ \\
\hline Superior Labral tear from Anterior to Posterior (SLAP tear) & A tear of the superior labrum at $11-2$ o'clock position \\
\hline GlenoLabral Articular Disruption (GLAD) & $\begin{array}{l}\text { The labroligamentous complex is partially teared and the cartilage is } \\
\text { damaged }\end{array}$ \\
\hline Capsular lesions & $\begin{array}{l}\text { A lesion to the shoulder joint capsule including the following lesions: } \\
\text { HAGL, GAGL, AIGHL, IGHL, PHAGL }\end{array}$ \\
\hline Humeral Avulsion of the Glenohumeral Ligament (HAGL) & $\begin{array}{l}\text { Avulsion fracture of the inferior glenohumeral ligament at the humeral } \\
\text { insertion }\end{array}$ \\
\hline Glenoid Avulsion of the Glenohumeral Ligament (GAGL) & $\begin{array}{l}\text { Avulsion fracture of the inferior glenohumeral ligament at the glenoid } \\
\text { insertion }\end{array}$ \\
\hline Anterior Inferior GlenoHumeral Ligament avulsion(AIGHL) & Avulsion fracture of the anterior inferior glenohumeral ligament \\
\hline Inferior GlenoHumeral Ligament avulsion (IGHL) & Avulsion fracture of the inferior glenohumeral ligament \\
\hline $\begin{array}{l}\text { Posterior Humeral Avulsion of the Glenohumeral Ligament } \\
\text { (PHAGL) }\end{array}$ & $\begin{array}{l}\text { Avulsion fracture of the posterior inferior glenohumeral ligament at the } \\
\text { humeral insertion }\end{array}$ \\
\hline Rotator-cuff tear & $\begin{array}{l}\text { A tear of the } \mathrm{m} \text {. Subscapularis, } \mathrm{m} \text {. Infraspinatus, } \mathrm{m} \text {. Supraspinatus or } \mathrm{m} \text {. } \\
\text { Teres minor }\end{array}$ \\
\hline Chondral lesion & Chondral injury of the glenoid or humeral head \\
\hline Long head of the biceps tear & (Partial) tear of the long head of the biceps \\
\hline
\end{tabular}


of the included articles may not have used the same definitions. Secondary outcomes include the number of dislocations, whether patients experienced a complete dislocation or subluxation and whether patients experienced a recurrent dislocation $(\geq 2)$ or first-time dislocation. A dislocation was defined as any type of anterior dislocation, including subluxations and complete dislocations. A complete dislocation was defined as an anterior dislocation requiring manual reduction. All data were extracted and transferred by 1 Author (C.R.) to Excel (Microsoft Corporation. Microsoft Excel [Internet]. 2012. Available from: https://office.micro soft.com/excel). To evaluate the reliability and completeness of the data extracted by C.R., the data from a random sample of 10 articles were independently extracted and transferred by Author 2 (L.P.E.V.) to an independent Excel database. The two databases were compared by both authors. Both databases corresponded with each other.

\section{Statistical analysis}

Baseline characteristics and outcome data were presented using descriptive statistics reported as mean and SD. In case the SD was not reported, it was estimated using the sample size and range according to Walter et al.[33]. The data were pooled and a weighted mean was calculated. Proportions of lesions were analyzed for the amount of dislocations $(1, \geq 2)$ and type of dislocation (complete dislocation, subluxation). Other outcomes, such as modality, age, patient characteristics and indication, could not be analyzed because there were not enough data. Instead, these outcomes were described. When the prevalence of a lesion was not reported in an article, it did not take part in the analysis. Proportions were compared with chi-square tests. Confidence intervals (CI) were reported where possible. Odds-ratios (ORs) and 95\% CI were calculated with Review Manager version 5.3 (the Nordic Cochrane Center, Copenhagen, Denmark). $P$ values $\leq 0.05$ were considered significant. Heterogeneity between studies was determined by the $I^{2}$ value. $I^{2}>50 \%$ was considered to signify substantial heterogeneity [34].

\section{Results}

\section{Screening and study characteristics}

After duplicates were removed, 6662 studies were screened by title and abstract. The full-text of 79 articles was read to determine if the article was eligible for inclusion. The search strategy resulted in 22 articles meeting the inclusion criteria. Reasons for exclusion during full-text screening are shown in Fig. 1.

Seven studies had a prospective design and 15 studies had a retrospective design (Additional file 2: Table 1).
Arthroscopy was the modality of choice in 15 studies. The highest ranking modality was MRI or MRA in four studies and CT in two studies. One MRI study identified soft-tissue and bony lesions while the other three identified only softtissue lesion. Both CT studies identified only bony lesions. Athletes were studied in three articles and military personnel was studied in two articles. A population older than 35 was studied in two articles. The indication for inclusion was surgery in 13 articles, diagnostic imaging in six articles, a dislocation event in two articles and diagnostic arthroscopy in one article.

A total of 1920 shoulders were studied for lesions associated with traumatic anterior shoulder dislocation. The pooled mean age was $26.2 \pm 8.9$ years. There were $581 \mathrm{com}$ plete dislocations from 6 studies, 101 subluxations from 3 studies, 1152 recurrent dislocations from 15 studies and 392 first-time dislocations from 10 studies. For every dislocation, 1.8 lesions were identified. Lesions were determined using arthroscopy in $72 \%$ of shoulders and imaging in $28 \%$ of shoulders.

\section{Quality control}

Four articles had one question of the JBI critical appraisal answered with 'No'. All other questions were answered with 'Yes'. Question 1, regarding the appropriateness of the studied sample was answered with 'No' for Takase et al. Their objective was to investigate lesions in all shoulders; however, they only included patients undergoing Bankart repair. Question 4, regarding how detailed the study setting was described was answered with 'No' for Ozaki et al. Their methods describe the presence of Hill-Sachs as inclusion criteria, however from the results can be concluded that a general population was studied, including without Hill-Sachs. Question 7, regarding the reliability of measurements and standardized methods for all patients was answered with 'No' for Owens et al. and Kim et al. Both studies diagnosed part of their population by arthroscopy and part by imaging (Additional file 2: Table 2). Two authors (C.R. and L.P.E.V.) completed the JBI critical appraisal independently of each other. Minor differences were found which were resolved by discussion and consensus.

\section{Prevalence of bony lesions}

In a pooled sample of 1,845 shoulders from 22 studies, a bony lesion was reported in 96\%. A Hill-Sachs (69\%) lesion was most prevalent and bony Bankart (13\%) least prevalent (Table 2). The lesion prevalence found in each individual study was summarized in Additional file 2: Table 3. 
Fig. 1 PRISMA Flow diagram[60]

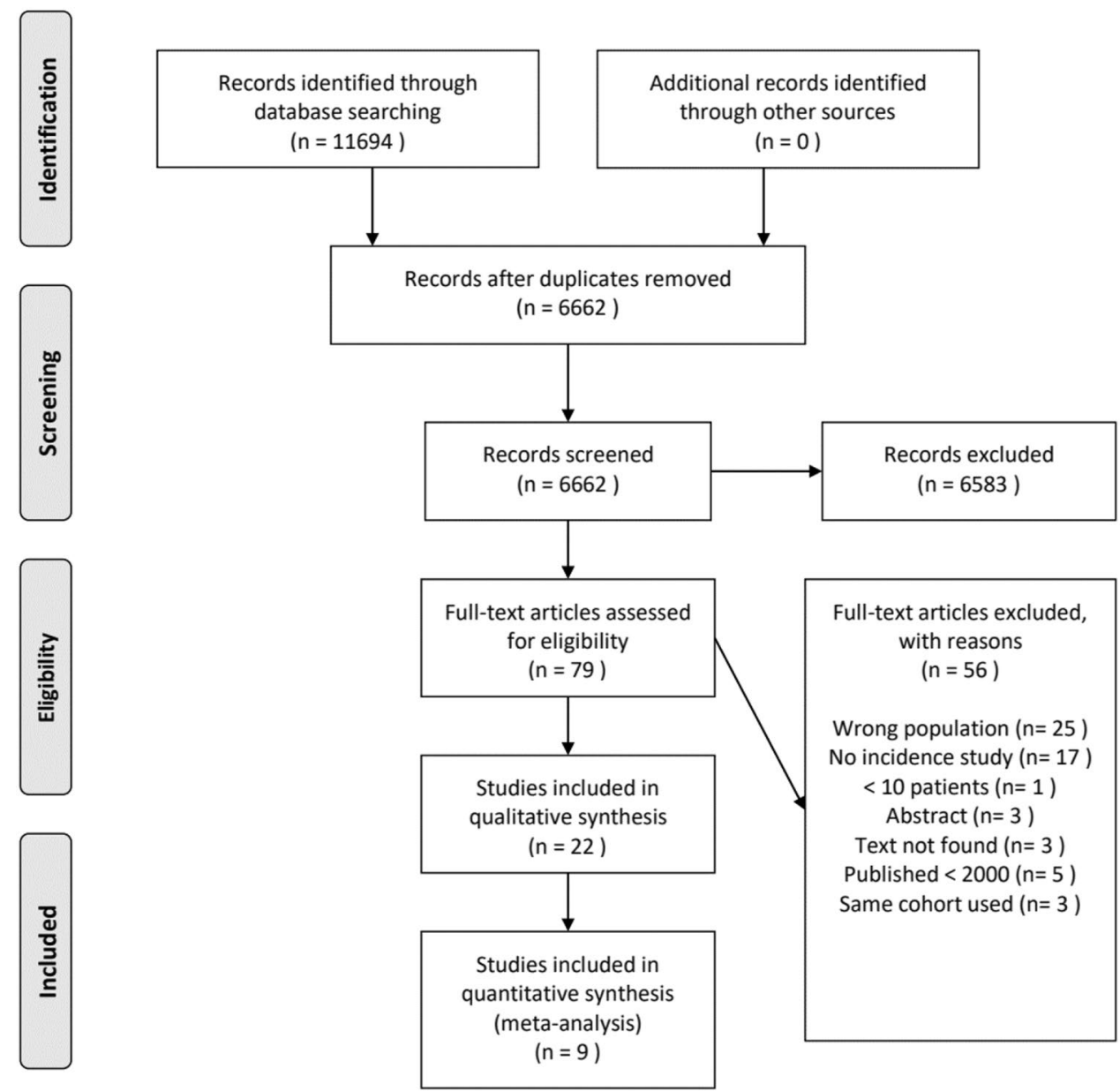

Table 3 Prevalence of labral lesions

Table 2 Prevalence of bony lesions

\begin{tabular}{llllc}
\hline & Studies $(n)$ & Shoulder $(n)$ & $\begin{array}{l}\text { Preva- } \\
\text { lence } \\
(\%)\end{array}$ & Range (\%) \\
\hline Hill-Sachs & 20 & 1731 & 69 & $13-100$ \\
Bony glenoid & 10 & 983 & 37 & $6-86$ \\
Loose body & 7 & 566 & 15 & $9-44$ \\
Bony Bankart & 8 & 889 & 13 & $0-43$ \\
\hline
\end{tabular}

\section{Prevalence of soft-tissue lesions}

In a pooled sample of 1320 shoulders from 17 studies, a labral lesion was reported in $97 \%$ of shoulders. A Bankart (67\%) lesion was most prevalent, followed by posterior Bankart (23\%) and SLAP (23\%) lesions (Table 3). A Perthes lesion was least prevalent (14\%).

In a pooled sample of 1018 shoulders from 11 studies, a capsular lesion was reported in 16\% (Table 4).

In a pooled sample of 1634 shoulders from 19 studies, there were 1.3 soft-tissue lesions per shoulder. Excluding labral lesions, a rotator-cuff tear (17\%) was most prevalent

\begin{tabular}{llllc}
\hline & Studies $(n)$ & Shoulder $(n)$ & $\begin{array}{l}\text { Preva- } \\
\text { lence } \\
(\%)\end{array}$ & Range (\%) \\
\hline Bankart & 14 & 993 & 67 & $20-100$ \\
Posterior Bankart & 3 & 204 & 23 & $3-42$ \\
Perthes & 3 & 79 & 14 & $0-32$ \\
ALPSA & 8 & 542 & 18 & $0-26$ \\
SLAP & 15 & 1,245 & 23 & $0-64$ \\
GLAD & 4 & 355 & 4 & $0-20$ \\
\hline
\end{tabular}

Table 4 Prevalence of capsular lesions

\begin{tabular}{lllll}
\hline & Studies $(n)$ & Shoulder $(n)$ & $\begin{array}{l}\text { Preva- } \\
\text { lence }(\%)\end{array}$ & Range (\%) \\
\hline HAGL & 10 & 988 & 3 & $1-21$ \\
AIGHL & 1 & 30 & 90 & N.a \\
IGHL & 1 & 42 & 33 & N.a \\
PHAGL & 1 & 42 & 31 & N.a \\
GAGL & 1 & 25 & 40 & N.a \\
\hline
\end{tabular}


Table 5 Prevalence of soft-tissue lesions

\begin{tabular}{lllll}
\hline & Studies $(n)$ & Shoulder $(n)$ & $\begin{array}{l}\text { Preva- } \\
\text { lence } \\
(\%)\end{array}$ & Range (\%) \\
\hline Rotator-cuff tear & 13 & 1290 & 17 & $2-64$ \\
$\begin{array}{l}\text { Chondral } \\
\begin{array}{l}\text { Long head of the } \\
\text { biceps }\end{array}\end{array}$ & 3 & 325 & 9 & $4-28$ \\
\hline
\end{tabular}

(Table 5). A long head of the biceps lesion was least prevalent. $(8 \%)$.

\section{Meta-analyses of lesion prevalence for first-time and recurrent dislocations}

A lower proportion of Hill-Sachs lesions was observed following first-time dislocation (71\%, range 58-83\%) compared to recurrent dislocation $(85 \%$, range $70-95 \% ; P=0.01$, $I^{2}=71 \%$; Fig. 2).

A lower proportion of Bankart lesions was observed following first-time dislocation (59\%, range 18-100\%) compared to recurrent dislocation $(66 \%$, range $28-100 \%$; $P=0.05, I^{2}=46 \%$; Fig. 3 ).

No significant difference was observed for SLAP lesions following first-time dislocation (27\%, range 16-45\%) compared to recurrent dislocation (28\%, range $20-45 \%$; $P=$ n.s., $I^{2}=0 \%$; Fig. 4 ).

No significant difference was observed for partial- or full thickness rotator-cuff tears following first-time dislocation (7\%, range $0-12 \%$ ) compared to recurrent dislocation (14\%, range $3-20 \%$; $P=$ n.s., $I^{2}=0 \%$; Fig. 5).

No significant difference was observed for bony Bankart lesions following first-time dislocation (12\%, range 9-18\%) compared to recurrent dislocation (17\%, range $11-28 \%$; $P=$ n.s., $I^{2}=25 \%$; Fig. 6 ).

No significant difference was observed for HAGL lesions following first-time dislocation (4\%, range 0-9\%) compared to recurrent dislocation ( $1 \%$, range $0-4 \% ; P=$ n.s., $I^{2}=54 \%$; Fig. 7).

No significant difference was observed for ALPSA lesions following first-time dislocation (16\%, range $0-30 \%$ ) compared to recurrent dislocation (22\%, range $13-19 \%$; $P=$ n.s., $I^{2}=62 \%$; Fig. 8 ).

\section{Meta-analysis of lesions prevalence for subluxations and complete dislocations}

A lower proportion of Hill-Sachs lesions was observed following subluxation (54\%, range 43-60\%) compared to complete dislocation $\left(82 \%\right.$, range $65-86 \% ; P<0.01, I^{2}=35 \%$; Fig. 9).

\begin{tabular}{|c|c|c|c|c|c|c|c|c|c|c|c|}
\hline \multirow[b]{2}{*}{ Study or Subgroup } & \multicolumn{2}{|c|}{ First-time dislocation } & \multicolumn{2}{|c|}{ Recurrent dislocation } & \multicolumn{3}{|c|}{ Odds Ratio } & \multirow{2}{*}{\multicolumn{4}{|c|}{$\begin{array}{c}\text { Odds Ratio } \\
\mathrm{M}-\mathrm{H}, \text { Random, } 95 \% \mathrm{Cl}\end{array}$}} \\
\hline & Events & Total & Events & Total & Weight & M-H, Random, $95 \% \mathrm{Cl}$ & Year & & & & \\
\hline Widjaja 2006 & 10 & 15 & 32 & 46 & $13.2 \%$ & $0.88[0.25,3.03]$ & 2006 & & & & \\
\hline Yiannakopoulos 2007 & 15 & 23 & 97 & 104 & $13.9 \%$ & $0.14[0.04,0.43]$ & 2007 & & & & \\
\hline Kim 2010 (1) & 19 & 33 & 105 & 111 & $14.5 \%$ & $0.08[0.03,0.23]$ & 2010 & & & & \\
\hline Gutierrez 2012 & 37 & 45 & 48 & 51 & $12.1 \%$ & $0.29[0.07,1.17]$ & 2012 & & & & \\
\hline Ozaki 2014 & 25 & 30 & 42 & 48 & $12.9 \%$ & $0.71[0.20,2.58]$ & 2014 & & & & \\
\hline Shin 2016 & 24 & 33 & 72 & 89 & $15.7 \%$ & $0.63[0.25,1.60]$ & 2016 & & & & \\
\hline Rugg 2017 & 40 & 58 & 82 & 114 & $17.7 \%$ & $0.87[0.43,1.73]$ & 2017 & & & & \\
\hline Total $(95 \% \mathrm{Cl})$ & & 237 & & 563 & $100.0 \%$ & $0.38[0.18,0.81]$ & & & & & \\
\hline Total events & 170 & & 478 & & & & & & & & \\
\hline \multicolumn{8}{|c|}{$\begin{array}{l}\text { Heterogeneity: } \text { Tau }^{2}=0.69 ; \mathrm{Chi}^{2}=20.44, \mathrm{df}=6(P=0.002) ; I^{2}=71 \% \\
\text { Test for overall effect: } Z=2.52(P=0.01)\end{array}$} & 0.01 & $\begin{array}{l}0.1 \\
\text { Favours First-time dislo. }\end{array}$ & 1 Favours Rect & $\begin{array}{l}10 \\
\text { urrent dislo. }\end{array}$ \\
\hline
\end{tabular}

Fig. 2 Meta-analysis of Hill-Sachs first-time vs recurrent proportions. This meta-analysis shows the odds ratio for studies $(n=7)$ that reported the prevalence of Hill-Sachs lesions in first-time dislocations compared to recurrent dislocations

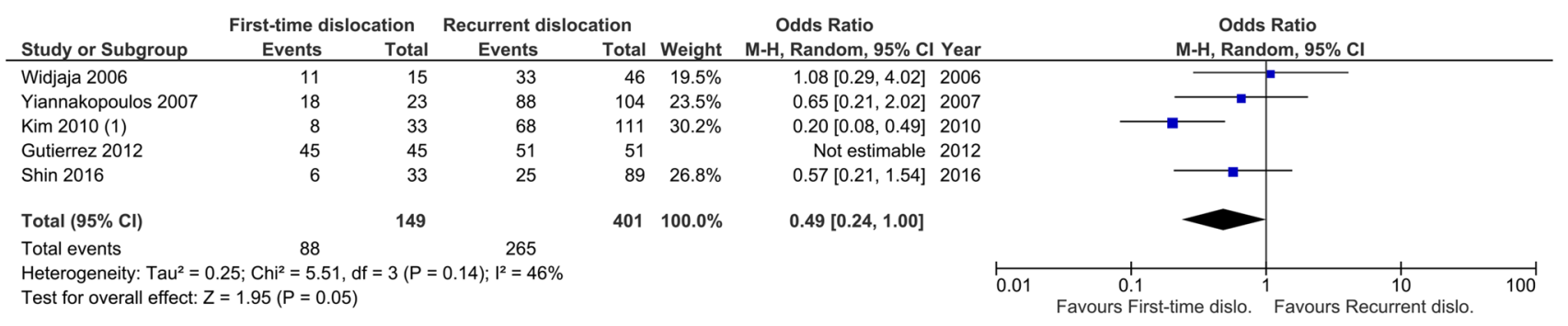

Fig. 3 Meta-analysis of Bankart first-time vs recurrent dislocation proportions. This meta-analysis shows the odds ratio for studies $(n=5)$ that reported the prevalence of Bankart lesions in first-time dislocations compared to recurrent dislocations 


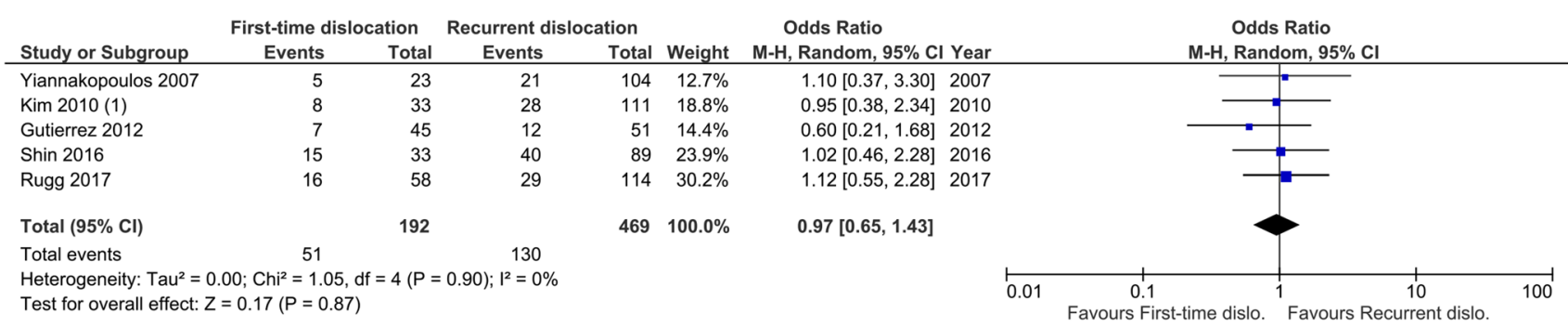

Fig. 4 Meta-analysis of SLAP first-time vs recurrent dislocation proportions. This meta-analysis shows the odds ratio for studies $(n=5)$ that report the prevalence of SLAP lesions in first-time dislocations compared to recurrent dislocations

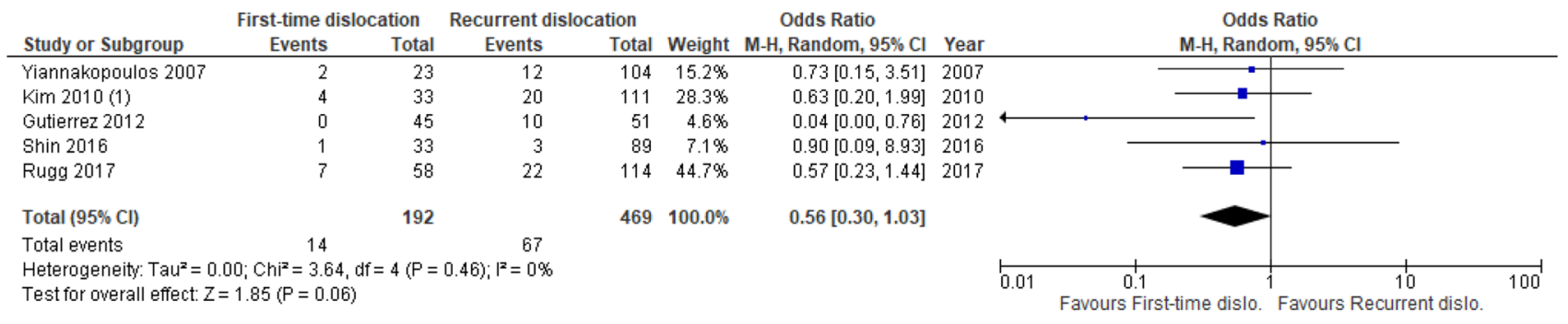

Fig. 5 Meta-analysis of rotator-cuff tear first-time vs recurrent dislocation proportions. This meta-analysis shows the odds ratio for studies $(n=5)$ that report the prevalence of rotator-cuff tears in first-time dislocations compared to recurrent dislocations

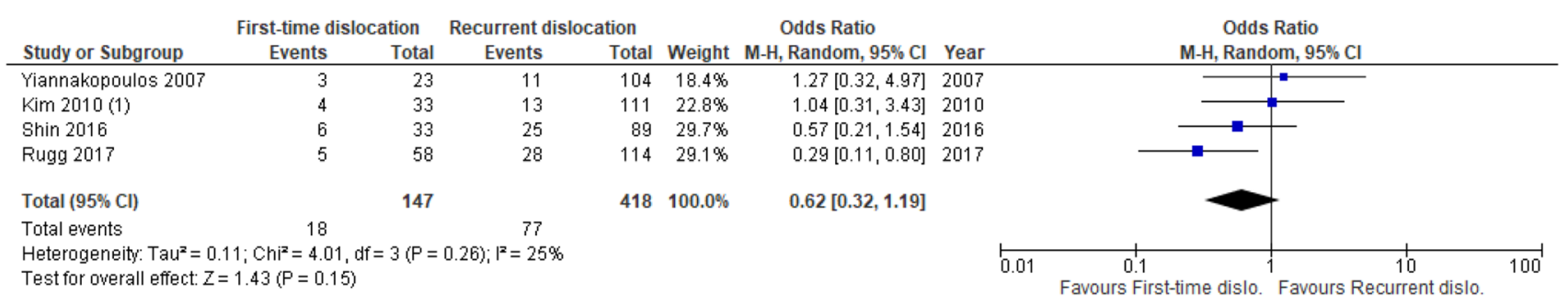

Fig. 6 Meta-analysis of bony Bankart first-time vs recurrent dislocation proportions. This meta-analysis shows the odds ratio for studies $(n=4)$ that report the prevalence of bony Bankart lesions in first-time dislocations compared to recurrent dislocations

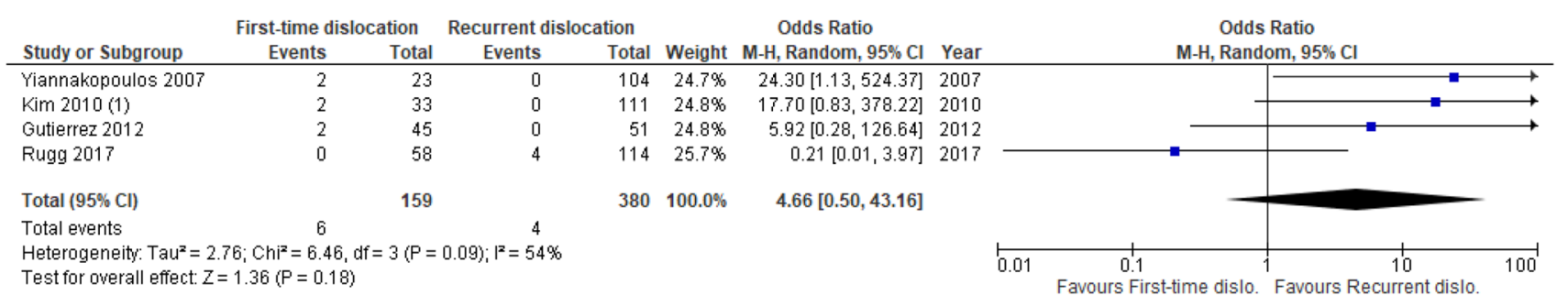

Fig. 7 Meta-analysis of HAGL first-time vs recurrent dislocation proportions. This meta-analysis shows the odds ratio for studies $(n=4)$ that report the prevalence of HAGL lesions in first-time dislocations compared to recurrent dislocations 


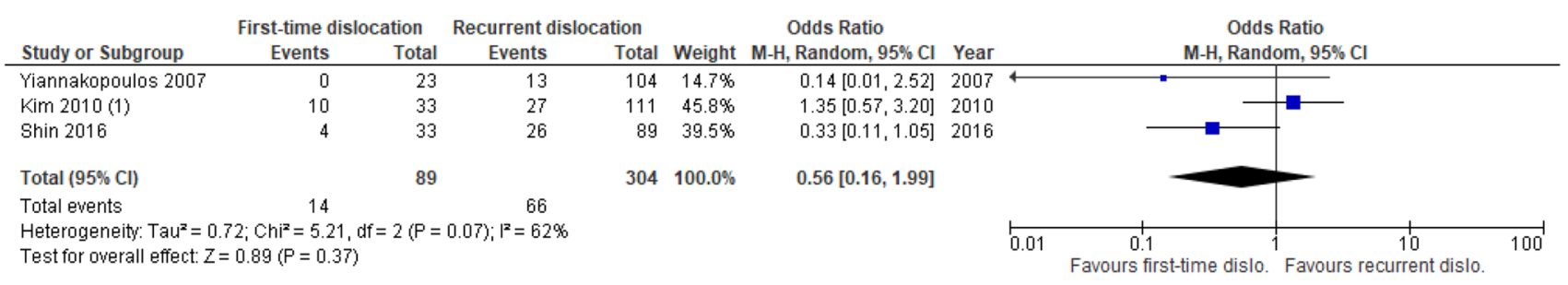

Fig. 8 Proportions of ALPSA lesions for first-time dislocations compared to recurrent dislocations. This meta-analysis shows the odds ratio for studies $(n=3)$ that report the prevalence of ALPSA lesions in first-time dislocations compared to recurrent dislocations

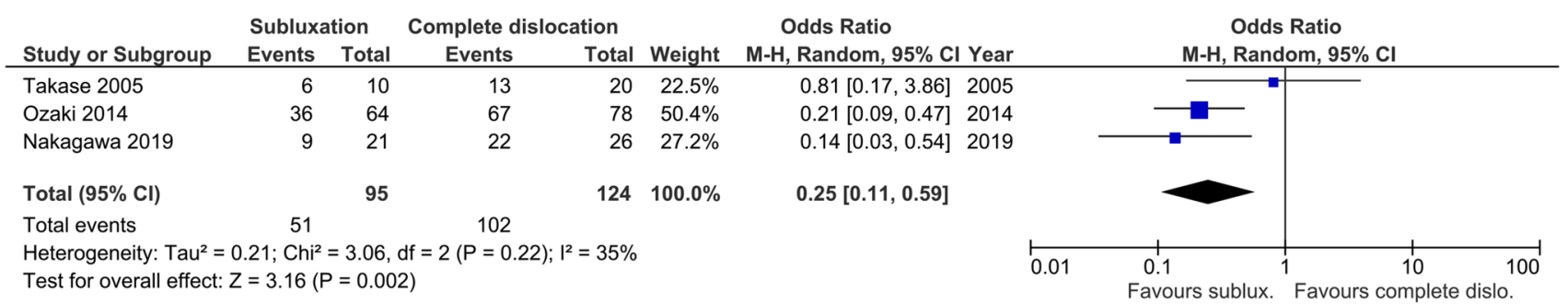

Fig. 9 Meta-analysis of Hill-Sachs subluxation vs complete dislocation proportions. This meta-analysis shows the odds ratio for studies $(n=3)$ that report the prevalence of Hill-Sachs lesions in sub-dislocations compared to complete dislocations

\section{Discussion}

The most important finding of the present study was that Hill-Sachs and Bankart lesions were found in higher proportions in recurrent dislocations compared to first-time dislocations. The prevalence of bony Bankart, HAGL, SLAP, rotator-cuff tear and ALPSA were similar when comparing recurrent- and first-time dislocations, suggesting these lesions may be more common after first-time dislocation than previously thought. However, results should be interpreted with caution due to the large range in prevalence; heterogeneity found in the Hill-Sachs, HAGL and ALPSA analyses; and limited amount of studies reporting on capsular lesions.

Current data may be valuable for health professionals in the outpatient clinic with regard to shared decision-making. The results may assist in estimating the risk of new lesions after recurrence, especially as literature on this topic is currently lacking. When Hill-Sachs and Bankart lesions are present, current results suggest that no other lesions may be expected after recurrence. Also, as the optimal management may differ between lesions, the epidemiological results may assist in anticipating which lesion is likely present and which type of surgical procedure may be indicated [35-39]. For optimal shared decision-making, the amount of dislocations and whether the dislocation was complete or not should be taken into consideration.

The initial size, location and the subsequent increase of a bony lesion after a recurrent dislocation may be an important factor in surgical decision-making. Hasegawa et al. found the number of dislocation events to be a predictor of the amount of bipolar bone loss [40]. A correlation between bony lesion size and risk of recurrence has been described by multiple authors and literature suggests early surgical intervention to decrease risk of recurrence and increase return-to-play [41, 42]. Current results may confirm this conclusion. Untreated traumatic rotator-cuff tears may cause more pain and dysfunction over time and together with capsulolabral damage may lead to increased instability [20]. Individual capsulolabral and HAGL lesions are also thought to play a role in glenohumeral instability [43-45]. The hypothesis that lesions increase in size after recurrence rather than in prevalence is supported by current results and literature. Literature suggests that instability increases over time in untreated shoulders even though current results suggest that most lesions may not increase in prevalence after recurrence. Health professionals may therefore need to consider early surgical stabilization after first-time dislocation to limit the increase in size of these lesions.

A varying prevalence was found between studies for most lesions. Challenges in classifying, measuring and defining lesions may attribute to this. The glenoid track method is used to determine the extent of glenoid bone loss but lacks a standardized method to produce the image, causing challenges with regard to reproducibility and the reliability [46]. It may be difficult to identify lesions of the labrum correctly, such as Bankart, as literature shows discrepancies in defining lesions according to the clockface-method [47, 48]. The Snyder classification is used to evaluate SLAP lesions but 
shows high inter-observer and intra-observer variability, even between experience specialists [49]. Lastly, the Outerbridge classification is used for the classification of chondral lesions but studied only limited times in small populations [50]. Extensions or revision of current classification systems may assist in better identification and evaluation of lesions, as was done by Maffet et al. for the SLAP lesion [51]. The varying prevalence may also be explained by demographical differences. This includes sports that are more popular in certain countries but also the glenoid morphology, which may differ between populations [52].

The term subluxation presents difficulties in interpretation as a clear method of identifying subluxations in practice is currently lacking. One can even question whether a Hill-Sachs lesion can be caused by subluxation as the (postero-superior) humeral head may not impact against the glenoid. When Hill-Sachs is present following subluxation the displacement may have been such that one should speak of a complete dislocation, perhaps with spontaneous reduction. Current literature suggests that false identification of Hill-Sachs lesions may be a result of normal ossification of the humeral head in developing shoulders, possibly explaining Hill-Sachs lesions following subluxation [53]. It is interesting that some studies identified posterior Bankart lesions in patients suffering from anterior dislocation. A systematic review by Ernat et al. discussed $270^{\circ}$ and $360^{\circ}$ arthroscopic repair in a group of patients who suffered from an isolated anterior instability, suggesting that posterior lesions may occur after anterior dislocation [54].

The large range in prevalence found in this review may also be partly explained by the varying accuracy of diagnostic tools between studies. The sensitivity and specificity of MRA compared to the gold standard arthroscopy for Perthes was found to be $67 \%$ and $100 \%$, respectively, by Kehdr et al. and sensitivity was found to be $100 \%$ by Elkharbotly et al. [6, 8] Perthes and GLAD lesions may be difficult to diagnose because the labrum can remain in its natural position even though the labrum is torn $[47,55,56]$. Inexperienced assessors are thought to make less accurate and less reliable diagnoses, an effect that is enhanced for difficult to diagnose lesions, such as Perthes and GLAD $[57,58]$. Accuracy may be improved by taking notice of subtle MR intensity changes or placing the arm in the ABER position, although the latter is debated due to increased scan time and discomfort [56, 59].

The results of our study should be interpreted with caution in light of the following limitations. First, some lesions were reported in only a small amount of studies, increasing the risk of selection bias. The meta-analysis of the bony Bankart, HAGL and ALPSA only included four or less studies and the prevalence of the AIGHL, IGHL, PHAGL and GAGL were determined from one study. In case of these lesions, the data may not be sufficient to draw firm conclusions. Second, there is a risk of selection bias because most studies included patients undergoing surgical intervention. In a patient group not undergoing surgical intervention, there may be a lower frequency of lesions. Third, demographical differences may affect the prevalence of lesions due to high-risk sports being popular in certain countries or varying glenoid morphology between populations [52]. Fourth, not all lesions may have been identified in retrospective arthroscopy studies because the procedure may have had a different goal, causing the surgeon to pay less attention to uncommon lesions. These limitations may be reflected by the varying prevalence found between studies and substantial heterogeneity.

Future research should focus on multiple aspects. First, determining the role of lesion size and lesion growth in evaluating risk of recurrence, return-to-play and surgical decision-making. This includes soft-tissue lesions and the role of posterior lesions following anterior dislocation. Literature suggests there may be a correlation but high level evidence is currently lacking. Second, creating standardized methods to diagnose and classify radiological and symptomatic lesions and to define the term subluxation. This may lower the observed variance. Third, determine the sensitivity and specificity of diagnostic tools in large and representative populations as this is currently debated in the literature. Fourth, for both soft-tissue lesions and bony lesions, the aim should be to increase the accuracy of the diagnosis for MRI. It is especially important to improve bone MRI so the patient is not exposed to the radiation from CT. Fifth, study the prevalence of shoulder lesions following anterior dislocation in larger and more populations to increase the value of future systematic reviews like this one. Lastly, demographical variations of glenoid morphology between populations should be studied, also in currently less represented populations.

\section{Conclusion}

Higher proportions of Hill-Sachs and Bankart were observed in recurrent dislocations compared to first-time dislocations. No difference was observed for bony Bankart, HAGL, SLAP, rotator-cuff tear and ALPSA. Especially when a Hill-Sachs or Bankart is present after first-time dislocation, early surgical stabilization may need to be considered as other lesions may not be expected after recurrence and to limit lesion growth. However, results should be interpreted with caution due to substantial heterogeneity and large variance.

Supplementary Information The online version contains supplementary material available at https://doi.org/10.1007/s00167-021-06847-7.

Acknowledgements Not applicable. 
Author contribution CR contributed to all processes of the review and drafted the manuscript. LPEV contributed to the screening of studies, extracting data, statistical analysis and writing of the manuscript. SPV contributed to the writing of the search term and adapting the search to all databases. DFPD contributed to interpreting the results and writing the discussion from an orthopedic perspective. MM contributed to interpreting the results and writing the discussion from a radiological perspective. MPJB supervised the process and contributed to setting up the review, interpreting the results and writing the discussion.

Funding No funding or sponsorship was received.

\section{Declarations}

\section{Conflict of interest None.}

Ethical approval Not applicable.

Informed consent Not applicable.

Open Access This article is licensed under a Creative Commons Attribution 4.0 International License, which permits use, sharing, adaptation, distribution and reproduction in any medium or format, as long as you give appropriate credit to the original author(s) and the source, provide a link to the Creative Commons licence, and indicate if changes were made. The images or other third party material in this article are included in the article's Creative Commons licence, unless indicated otherwise in a credit line to the material. If material is not included in the article's Creative Commons licence and your intended use is not permitted by statutory regulation or exceeds the permitted use, you will need to obtain permission directly from the copyright holder. To view a copy of this licence, visit http://creativecommons.org/licenses/by/4.0/.

\section{References}

1. Khiami F, Gérometta A, Loriaut P (2015) Management of recent first-time anterior shoulder dislocations. Orthop Traumatol Surg Res 101:S51-57

2. Yeap JS, Lee DJ, Fazir M, Borhan TA, Kareem BA (2004) The epidemiology of shoulder dislocations in Malaysia. Med J Malays 59(Suppl F):19-23

3. Zacchilli MA, Owens BD (2010) Epidemiology of shoulder dislocations presenting to emergency departments in the United States. J Bone Jt Surg Am 92:542-549

4. Cibulas A, Leyva A, Cibulas G 2nd, Foss M, Boron A, Dennison J et al (2019) Acute shoulder injury. Radiol Clin N Am 57:883-896

5. Sedeek SM, Bin Abd Razak HR, Ee GWW, Tan AHC (2014) First-time anterior shoulder dislocations: should they be arthroscopically stabilised? Singap Med J 55:511-516

6. Elkharbotly A (2016) Shoulder joint instability evaluation by CT arthrography and MR arthrography. Egypt J Radiol Nucl Med 47:937-948

7. Kadi R, Milants A, Shahabpour M (2017) Shoulder anatomy and normal variants. J Belg Soc Radiol 101:3

8. Khedr SA, Kassem HM, Azab MA (2013) Comparative study of direct MR arthrography and CT arthrography with arthroscopic correlation in preoperative evaluation of anterior shoulder instability. Egypt J Radiol Nucl Med 44:817-827

9. Liu F, Cheng X, Dong J, Zhou D, Sun Q, Bai X et al (2019) Imaging modality for measuring the presence and extent of the labral lesions of the shoulder: a systematic review and meta-analysis. BMC Musculoskelet Disord 20:487

10. Liu YL, Cui GQ, Ao YF, Xiao J, Yan H, Yu CL (2007) Posterior and superior compression test and weight-bearing on elbow test for the diagnosis of superior labrum anterior to posterior lesion. Zhonghua wai ke za zhi [Chin J Surg] 45:1382-1384

11. Carrazzone OL, Tamaoki MJ, Ambra LF, Neto NA, Matsumoto MH, Belloti JC (2011) Prevalence of lesions associated with traumatic recurrent shoulder dislocation. Rev Bras Ortop 46:281-287

12. Karuppal R (2017) Magnetic resonance imaging arthrogram findings in recurrent dislocation of shoulder. J Evol Med 6:3452-3458

13. Owens BD, Nelson BJ, Duffey ML, Mountcastle SB, Taylor DC, Cameron KL et al (2010) Pathoanatomy of first-time, traumatic, anterior glenohumeral subluxation events. J Bone Jt Surg Am 92:1605-1611

14. Smith TO, Daniell H, Geere JA, Toms AP, Hing CB (2012) The diagnostic accuracy of MRI for the detection of partial- and full-thickness rotator cuff tears in adults. Magn Reson Imaging 30:336-346

15. Kirkley A, Litchfield R, Thain L, Spouge A (2003) Agreement between magnetic resonance imaging and arthroscopic evaluation of the shoulder joint in primary anterior dislocation of the shoulder. Clin J Sport Med 13:148-151

16. Saba L, De Filippo M (2016) MR arthrography evaluation in patients with traumatic anterior shoulder instability. J Orthop 14:73-76

17. Balg F, Boileau P (2007) The instability severity index score. A simple pre-operative score to select patients for arthroscopic or open shoulder stabilisation. J Bone Jt Surg Br 89:1470-1477

18. DeFroda S, Bokshan S, Stern E, Sullivan K, Owens BD (2017) Arthroscopic Bankart repair for the management of anterior shoulder instability: indications and outcomes. Curr Rev Musculoskelet Med 10:442-451

19. DeFroda SF, Goyal D, Patel N, Gupta N, Mulcahey MK (2018) Shoulder instability in the overhead athlete. Curr Sports Med Rep 17:308-314

20. Gombera MM, Sekiya JK (2014) Rotator cuff tear and glenohumeral instability: a systematic review. Clin Orthop Relat Res 472:2448-2456

21. Verweij LP, Baden DN, van der Zande JM, van den Bekerom MP (2020) Assessment and management of shoulder dislocation. BMJ 371:m4485

22. Verweij LPE, van Spanning SH, Grillo A, Kerkhoffs G, PriesterVink S, van Deurzen DFP et al (2021) Age, participation in competitive sports, bony lesions, ALPSA lesions, $>1$ preoperative dislocations, surgical delay and ISIS score $>3$ are risk factors for recurrence following arthroscopic Bankart repair: a systematic review and meta-analysis of 4584 shoulders. Knee Surg Sports Traumatol Arthrosc 29:4004-4014

23. Borges Migliavaca C, Stein C, Colpani V, Barker TH, Munn Z, Falavigna $\mathrm{M}$ et al (2020) How are systematic reviews of prevalence conducted? A methodological study. BMC Med Res Methodol 20:96

24. Munn Z, Moola S, Riitano D, Lisy K (2014) The development of a critical appraisal tool for use in systematic reviews addressing questions of prevalence. Int J Health Policy Manag 3:123-128

25. Olds M, Ellis R, Donaldson K, Parmar P, Kersten P (2015) Risk factors which predispose first-time traumatic anterior shoulder dislocations to recurrent instability in adults: a systematic review and meta-analysis. Br J Sports Med 49:913-922

26. Saccomanno MF, Fodale M, Capasso L, Cazzato G, Milano G (2013) Generalized joint laxity and multidirectional instability of the shoulder. Joints 1:171-179

27. Salomonsson B, von Heine A, Dahlborn M, Abbaszadegan H, Ahlström S, Dalén N et al (2010) Bony Bankart is a positive 
predictive factor after primary shoulder dislocation. Knee Surg Sports Traumatol Arthrosc 18:1425-1431

28. Boffano M, Mortera S, Piana R (2017) Management of the first episode of traumatic shoulder dislocation. EFORT Open Rev 2:35-40

29. Liberati A, Altman DG, Tetzlaff J, Mulrow C, Gøtzsche PC, Ioannidis JP et al (2009) The PRISMA statement for reporting systematic reviews and meta-analyses of studies that evaluate healthcare interventions: explanation and elaboration. BMJ 339:b2700

30. Ouzzani M, Hammady H, Fedorowicz Z, Elmagarmid A (2016) Rayyan-a web and mobile app for systematic reviews. Syst Rev $5: 210$

31. Maio M, Sarmento M, Moura N, Cartucho A (2019) How to measure a Hill-Sachs lesion: a systematic review. EFORT Open Rev 4:151-157

32. Munn Z, Moola S, Lisy K, Riitano D, Tufanaru C (2015) Methodological guidance for systematic reviews of observational epidemiological studies reporting prevalence and cumulative incidence data. Int J Evid Based Healthc 13:147-153

33. Walter SD, Yao X (2007) Effect sizes can be calculated for studies reporting ranges for outcome variables in systematic reviews. J Clin Epidemiol 60:849-852

34. Higgins JP, Thompson SG (2002) Quantifying heterogeneity in a meta-analysis. Stat Med 21:1539-1558

35. Arliani GG, Astur DdC, Cohen C, Ejnisman B, Andreoli CV, Pochini AC et al (2011) Surgical versus nonsurgical treatment in first traumatic anterior dislocation of the shoulder in athletes. Open Access J Sports Med 2:19-24

36. Cvetanovich GL, Gowd AK, Agarwalla A, Forsythe B, Romeo AA, Verma NN (2019) Trends in the management of isolated SLAP tears in the United States. Orthop J Sports Med 7:2325967119833997-2325967119833997

37. Fox JA, Sanchez A, Zajac TJ, Provencher MT (2017) Understanding the Hill-Sachs lesion in its role in patients with recurrent anterior shoulder instability. Curr Rev Musculoskelet Med 10:469-479

38. Hasebroock AW, Brinkman J, Foster L, Bowens JP (2019) Management of primary anterior shoulder dislocations: a narrative review. Sports Med 5:31-31

39. Marco SM, Lafuente J, Ibán MAR, Heredia JD (2017) Controversies in the surgical management of shoulder instability: associated soft tissue procedures. Open Orthop J 11:989-1000

40. Hasegawa Y, Kawasaki T, Nojiri S, Sobue S, Kaketa T, Gonda Y et al (2019) The number of injury events associated with the critical size of bipolar bone defects in rugby players with traumatic anterior shoulder instability. Am J Sports Med 47:2803-2808

41. Hurley ET, Manjunath AK, Bloom DA, Pauzenberger L, Mullett $\mathrm{H}$, Alaia MJ et al (2020) Arthroscopic Bankart repair versus conservative management for first-time traumatic anterior shoulder instability: a systematic review and meta-analysis. Arthroscopy 36:2526-2532

42. Watson S, Allen B, Grant JA (2016) A clinical review of returnto-play considerations after anterior shoulder dislocation. Sports Health 8:336-341

43. George MS, Khazzam M, Kuhn JE (2011) Humeral avulsion of glenohumeral ligaments. J Am Acad Orthop Surg 19:127-133

44. Lee BG, Cho NS, Rhee YG (2011) Anterior labroligamentous periosteal sleeve avulsion lesion in arthroscopic capsulolabral repair for anterior shoulder instability. Knee Surg Sports Traumatol Arthrosc 19:1563-1569
45. Van Blarcum GS, Svoboda SJ (2017) Glenohumeral instability related to special conditions: SLAP tears, pan-labral tears, and multidirectional instability. Sports Med Arthrosc Rev 25:e12-e17

46. Schneider AK, Hoy GA, Ek ET, Rotstein AH, Tate J, Taylor DM et al (2017) Interobserver and intraobserver variability of glenoid track measurements. J Shoulder Elbow Surg 26:573-579

47. De Coninck T, Ngai SS, Tafur M, Chung CB (2016) Imaging the glenoid labrum and labral tears. Radiographics 36:1628-1647

48. Itoigawa Y, Hooke AW, Sperling JW, Steinmann SP, Zhao KD, Itoi E et al (2020) Bankart repair alone in combined Bankart and superior labral anterior-posterior lesions preserves range of motion without compromising joint stability. JSES Int 4:63-67

49. Ahsan ZS, Hsu JE, Gee AO (2016) The snyder classification of superior labrum anterior and posterior (SLAP) lesions. Clin Orthop Relat Res 474:2075-2078

50. Slattery C, Kweon CY (2018) Classifications in brief: outerbridge classification of chondral lesions. Clin Orthop Relat Res 476:2101-2104

51. Maffet MW, Gartsman GM, Moseley B (1995) Superior labrumbiceps tendon complex lesions of the shoulder. Am J Sports Med 23:93-98

52. Sarı A, Dinçel YM, Günaydın B, Çetin MÜ, Özçaglayan Ö, Bilsel K (2020) Assessment of the glenoid morphology based on demographic data in the Turkish population. Biomed Res Int 2020:5736136

53. Kelly A, Heath MR, Amoroso EE, Mackie AT, Greditzer HGt, Owens BD, et al (2021) Normal humeral head ossification in pediatric and adolescent shoulders can mimic Hill-Sachs lesions: a magnetic resonance imaging-based study. J Pediatr Orthop. https://doi.org/10.1097/bpo.0000000000002017

54. Ernat JJ, Yheulon CG, Shaha JS (2020) Arthroscopic repair of 270- and 360-degree glenoid labrum tears: a systematic review. Arthroscopy 36:307-317

55. Sanders TG, Tirman PF, Linares R, Feller JF, Richardson R (1999) The glenolabral articular disruption lesion: MR arthrography with arthroscopic correlation. AJR Am J Roentgenol 172:171-175

56. Wischer TK, Bredella MA, Genant HK, Stoller DW, Bost FW, Tirman PFJ (2002) Perthes lesion (a variant of the Bankart lesion). AJR Am J Roentgenol 178:233-237

57. Jonas SC, Walton MJ, Sarangi PP (2012) Is MRA an unnecessary expense in the management of a clinically unstable shoulder? A comparison of MRA and arthroscopic findings in 90 patients. Acta Orthop 83:267-270

58. van Grinsven S, Hagenmaier F, van Loon CJ, van Gorp MJ, van Kints MJ, van Kampen A (2014) Does the experience level of the radiologist, assessment in consensus, or the addition of the abduction and external rotation view improve the diagnostic reproducibility and accuracy of MRA of the shoulder? Clin Radiol 69:1157-1164

59. Schreinemachers SA, van der Hulst VPM, Jaap Willems W, Bipat S, van der Woude H-J (2009) Is a single direct MR arthrography series in ABER position as accurate in detecting anteroinferior labroligamentous lesions as conventional MR arthography? Skelet Radiol 38:675-683

60. Moher D, Liberati A, Tetzlaff J, Altman DG (2009) Preferred reporting items for systematic reviews and meta-analyses: the PRISMA statement. PLoS Med 6:e1000097

Publisher's Note Springer Nature remains neutral with regard to jurisdictional claims in published maps and institutional affiliations. 